\title{
Análisis de la cobertura del tema de tabaco en medios impresos mexicanos
}

\author{
Silvia Elena Llaguno-Aguilar, ${ }^{(1,2)}$ Ana del Carmen Dorantes-Alonso, ${ }^{(1)}$ James F Thrasher, $\mathrm{PhD},{ }^{(1,3)}$ \\ Victor Villalobos, MPH, (I) John C Besley, PhD. ${ }^{(3,4)}$
}

\section{Llaguno-Aguilar SE, Dorantes-Alonso AC, Thrasher JF, Villalobos V, Besley JC. Análisis de la cobertura de tema de tabaco en medios impresos mexicanos. Salud Publica Mex 2008;50 supl 3:S348-S354.}

\section{Resumen}

Se estima la frecuencia y la forma en que los medios de comunicación presentan el tema de tabaco y las políticas públicas para su control. Se recopilaron y analizaron 660 notas sobre tabaco publicadas en 2007 en seis diarios mexicanos. Se observó un gran incremento de notas en el último semestre del año, debido a la discusión de la legislación local y federal. La mayoría de las notas fue a favor del control de tabaco $(49 \%)$ o neutra al respecto (4l\%). Los temas más presentes fueron: legislación (36\%), el humo de tabaco (28\%), economía (24\%) y empaquetado (17\%). Los actores mencionados fueron: instituciones gubernamentales (24\%), legisladores (2I\%), tabacaleras (I8\%), y organizaciones no gubernamentales nacionales (7\%) e internacionales (8\%). Se debe aprovechar la disposición de los medios de comunicación, generalmente a favor, o con una postura neutra, para reallizar actividades de abogacía de las políticas públicas para el control del tabaco.

Palabras clave: tabaco; medios de comunicación; políticas públicas; México
Llaguno-Aguilar SE, Dorantes-Alonso AC, Thrasher JF, Villalobos V, Besley JC.

Analysis of coverage of the tobacco issue in Mexican print media.

Salud Publica Mex 2008;50 suppl 3:S348-S354.

\section{Abstract}

Estimate the frequency and manner in which mass media represent tobacco and tobacco control policies. 660 stories published in six Mexican newspapers during 2007 were content analyzed. The majority of the news stories were published in the last quarter of the year, due to discussion of pending local and federal legislation. The vast majority of the stories were in favor of tobacco control policies (49\%) or neutral (4I\%). The most frequently occurring themes in the stories were: legislation (36\%), tobacco smoke (28\%), economy (24\%), and packaging (17\%). Actors mentioned were: governmental institutions (24\%), legislators (21\%), tobacco industry (18\%), and national and international non-governmental organizations ( 7 and $8 \%$, respectively). Advocacy efforts should take advantage of mass media, which appears generally neutral or in favor of tobacco control policies in Mexico.

Key words: tobacco; mass media; policy; Mexico

vor. Por otra parte, los sistemas mediáticos más abiertos, como se ve actualmente en México, ${ }^{2}$ dan entrada a otros actores de la sociedad civil para transmitir las ideas y los argumentos que promueven sus propios intereses. En

(I) Departamento de Investigación sobre Tabaco, Instituto Nacional de Salud Pública, México.

(2) Genes, Mujeres ProSalud AC, México.

(3) Departamento de Promoción de la Salud, Escuela de Salud Pública, Universidad de Carolina del Sur, EUA.

(4) Health Promotion, Education and Behavior, School of Public Health, Universidad de Carolina del Sur, EUA.

Fecha de aceptado: 28 de abril de 2008

Solicitud de sobretiros: James F. Thrasher. Health Promotion, Education and Behavior, 800 Sumter Street Rum \#215, School of Public Health, University of South Carolina, Columbia, Sc 29208, USA

Correo electrónico: thrasher@qwm.sc.edu 
un contexto democrático, se reconoce que los medios de comunicación pueden influir en las políticas públicas, impulsando la agenda, los argumentos y la importancia de un cambio legislativo., ${ }^{3,4}$ La influencia de los medios puede ser directa -hacia los tomadores de decisiones- 0 indirecta -hacia la población en general- propiciando cambios en la opinión pública y las conductas relacionadas con el tema de interés.

La abogacía en medios de comunicación consiste en una serie de métodos y prácticas para promover la transformación de las políticas públicas sobre algún tema en particular, mediante la cobertura del mismo en los medios de comunicación. ${ }^{5,6}$ Para lograrlo, la abogacía puede recurrir a un amplio abanico de actividades para llamar la atención de los medios y dirigir los argumentos a favor de un cambio legislativo al grupo blanco, desde las conferencias de prensa y el cultivo de relaciones con los periodistas y reporteros hasta promover manifestaciones y otros eventos espectaculares. ${ }^{7}$

Hay evidencia de que los medios de comunicación, y la abogacía a través de ellos, influyen en las políticas públicas. Por ejemplo, un análisis longitudinal de 25 años en Canadá indicó que los medios impresos tuvieron más impacto en la adopción de leyes que promovieron áreas libres de humo de tabaco, que la investigación científica o el discurso político. ${ }^{8}$ El nivel de cobertura mediática de tabaco en comunidades estadounidenses se asocia con un mayor conocimiento de los daños por fumar, a la mayor desaprobación del fumar, menor prevalencia percibida del hábito de fumar en pares y menor prevalencia del fumar en jóvenes. ${ }^{9}$ Además, la abogacía en medios ha aumentado las notas sobre el tabaquismo, ${ }^{10}$ y la mayor frecuencia de notas, resultado de abogacía, se asocia con cambios en las políticas públicas en el ámbito local. ${ }^{11}$ La abogacía en medios de comunicación se ha convertido en una herramienta poderosa para promover las políticas públicas en contra de tabaquismo.

Para que la abogacía en medios de comunicación sea exitosa, debe reconocer el discurso mediático sobre el tema de interés e introducirlo con los argumentos más oportunos. ${ }^{12}$ Para ello es necesario estudiar la representación del tabaquismo en los medios impresos, como se ha hecho en Estados Unidos, ${ }^{13,14}$ Canadá ${ }^{8}$ y Australia. ${ }^{15,16}$ Los resultados indican que el tema de tabaco tiene buena cobertura en los medios de aquellos países, ${ }^{14}$ aunque el tema aparece más difuso en Estados Unidos que en Australia, ${ }^{16}$ y los mensajes de los activistas para el control del tabaco tienen menor coherencia que los mensajes de las tabacaleras y sus aliados. ${ }^{13}$ Se desconocen estudios de este tipo en otros países.

El presente es un informe sobre el monitoreo del tema de tabaco en seis medios impresos de México, durante 2007. Este documento aporta información sobre dicho tema en un país en vías de desarrollo, y podría ser insumo para los futuros esfuerzos mediáticos de los y las activistas mexicanas.

\section{Material y métodos}

Selección de medios: se realizó un seguimiento del tema de tabaco en medios impresos (diarios) del 1 de enero al 31 de diciembre de 2007. Fueron seleccionados seis diarios de acuerdo con lo significativo de su circulación y su línea editorial; cinco de ellos de circulación nacional (La Jornada 107 659; El Universal 81534 lunes a sábado, 161911 el domingo; Milenio Diario 61 855; Reforma 145 650; El Financiero 91 923), y uno de circulación en el Distrito Federal (DF) y su área metropolitana ( $\mathrm{La}$ Prensa 244 693). ${ }^{17}$ Para hacer el monitoreo se contrató a una empresa dedicada a la recopilación y análisis de información difundida en los medios de comunicación. Dicha empresa realizó la búsqueda en los diarios con base en las palabras clave: tabaco, tabaquismo, fumadores, fumar, cigarros y cigarrillos.

Sistema de codificación: como punto de partida se utilizó un sistema de codificación desarrollado para notas sobre el tema de tabaco en Estados Unidos. ${ }^{18}$ Posteriormente se redujo el grado de complejidad para aumentar el grado de fiabilidad entre las codificadoras para la aplicación independiente de cada código (alfa de Krippendorf ${ }^{19}$ $>=0.7$ ). Los códigos del sistema incluyen las características del texto periodístico (por ejemplo, el tipo de texto: nota, artículo, editorial, columna); la sección del diario donde se publicó la nota; los actores citados en el texto periodístico (institución gubernamental; legisladores; universidad, académicos y/o centros de investigación; industria tabacalera; organización no gubernamental (ONG) mexicana; organización no gubernamental internacional; otras empresas y empresarios); y el contenido de la nota (por ejemplo, legislación, economía, humo de tabaco, empaquetado del producto). El cuadro I muestra los temas de interés para el presente artículo y sus definiciones.

Analisis: cada nota fue archivada como documento de texto y analizada usando el software ATLAS.ti V5.2. La codificación la llevaron a cabo dos personas, aplicando los códigos de contenido a cada párrafo. Dentro del sistema utilizado, una nota o fragmento puede tener varios códigos y un código puede aparecer varias veces en una misma nota. Para este artículo, se tomó en cuenta solamente si el código aparecía o no dentro de la nota, sin contar el número de apariciones. Se asignó la tendencia tomando en cuenta el tono de la nota completa.

\section{Resultados}




\section{Cuadro I \\ Temas de la codificación en las notas sobre tabaco, México, 2007}

Tema

$\begin{array}{ll} & \text { Regulación y políticas públicas para el control del tabaco sólo en México (si fuera en otro país, usar evidencias de otros } \\ \text { países). Puede ser la política como tema general, pero es más probable que se mencione en el contexto de otro tema de } \\ \text { interés. Por ejemplo: I) las restricciones de fumar y el humo del tabaco; } 2 \text { ) las advertencias sanitarias y los componentes } \\ \text { Políticas de control de } & \begin{array}{l}\text { del tabaco; 3) la prohibición de publicidad y la publicidad, promoción y patrocinio. También se contemplan los acuerdos } \\ \text { nacionales (por ejemplo, 2004 Convenio entre la Secretaría de Salud y las tabacaleras) e internacionales (Convenio Marco } \\ \text { para el Control del Tabaco). }\end{array}\end{array}$

Humo de tabaco

El efecto del humo de tabaco sobre terceros (fumadores pasivos) y las restricciones para fumar. Si hay mención de áreas libres de humo, también implica el código de política siempre y cuando hable de México.

Economía

Costos del consumo de tabaco, en el ámbito social (p. ej., gasto en enfermedades atribuibles al tabaco, días que los fumadores no trabajan) e individual (p. ej., gasto en cigarros en la vida). Se consideran los impuestos y el impacto de los impuestos en el consumo. Se excluye el precio del cultivo de tabaco (usar códigos "Cultivo y Comercio").

Componentes y Componentes físicos o químicos del producto (p. ej., alquitrán, nicotina, amoniaco), el empaquetado del tabaco (p. ej., empaquetado del producto advertencias sanitarias, sea cual sea la leyenda).
La publicidad (p. ej., anuncios espectaculares, revistas, periódicos, internet, carteles en la calle), las promociones (cupones, muestras gratuitas de cigarros, ropa y artículos con logotipos) y el patrocino (de eventos deportivos, fiestas, conciertos, eventos artísticos) que tiene fin de promover el consumo del cigarro o la buena imagen de las tabacaleras.
Publicidad, promoción y patrocinio del tabaco
Tendencia positiva: A favor de las políticas públicas para el control del tabaco y en contra del consumo de tabaco. Tendencia negativa: En contra de las políticas públicas y a favor del consumo de tabaco.

Tendencia neutra: Presenta las perspectivas de manera balanceada, sin estar a favor ni en contra del tabaquismo o las políticas públicas
Evidencias mexicanas sobre tabaco
Evidencias y datos sobre el tabaco de investigaciones científicas en México y la población mexicana.
Evidencias de otros países sobre tabaco y su regulación
Evidencias y datos sobre el tabaco de investigaciones científicas realizadas en otros países, incluso las políticas públicas en ellos.
Para todo 2007, se recabaron 674 notas, de las cuales 14 no fueron válidas para el análisis, por no referirse al tema. El volumen mayor de notas (73\%) se obtuvo de tres diarios: El Universal $(n=187)$, Reforma $(n=174)$ y Milenio Diario $(n=127)$; el resto fueron de La Jornada $(n=91)$, El Financiero $(n=49)$ y La Prensa $(n=46)$. Sobre la tendencia, el rango de notas de tendencia negativa fue entre 4\% (2 de 45 en La Prensa) y 13\% (16 de 124 en Milenio Diario). El Financiero, diario especializado en finanzas y economía, tuvo el menor porcentaje de notas positivas ( $29 \%$ o 14 de 48 ) y el mayor porcentaje de notas neutras (63\% o 30 de 48 ). Los demás diarios tuvieron un porcentaje similar de notas con tendencia positiva (rango de 45 a 64\%) y de tendencia neutra (rango de 31 a $48 \%$ ).

La figura 1 muestra el total de notas por mes y la tendencia de cada una de ellas. En el primer semestre del año, destaca un pico de notas publicadas en mayo, debido a que en ese mes se celebra el Día Mundial sin Tabaco, una fecha promovida por la Organización Mundial de la Salud (OMS) para llamar atención sobre el tema de tabaco. Mayo fue el mes con el mayor porcentaje de notas de tendencia positiva (73\%) en todo 2007. En septiembre se sobrepasó el pico de mayo, cuando se empezó a discutir la nueva legislación para el control del tabaco, en los ámbitos local (DF) y federal. Los meses durante los cuales se discutieron las leyes tuvieron aproximadamente $48 \%$ (186 de 386) de notas positivas y $11 \%$ de notas negativas. A lo largo del periodo analizado, casi la mitad de las notas presentó una tendencia positiva (49\%), mientras $41 \%$ tuvo tendencia neutra y $10 \%$ tendencia negativa. La máxima proporción de notas con tendencia negativa fue de aproximadamente $20 \%$, en enero de 2007, cuando se publicaron solamente 15 
notas sobre tabaco.

En las notas sobre tabaco aparecieron varios temas. El más frecuente fue la legislación $(36 \%)$, seguido de humo de tabaco (28\%), economía (24\%), empaquetado $(17 \%)$ y la publicidad, promoción y patrocinio del tabaco (9\%). En la figura 2 se indican los momentos en que aparecieron estos temas por mes. En el pico de mayo sobresalió tanto la legislación como el humo de tabaco y el empaquetado. Mientras que esto temas bajaron en el tercer trimestre del año, las notas sobre economía subieron por la discusión sobre los nuevos gravámenes al tabaco y el uso de los ingresos al fisco que darían las entidades federativas a estos recursos. En el último trimestre se incrementaron las notas que hablaron sobre la legislación, porque en estos meses se dio simultáneamente la discusión de la ampliación de la Ley de Protección a la Salud de los No Fumadores en el DF y la de la Ley General para el Control del Tabaco. Se encontró también un incremento menor en las notas que abordaron los temas contemplados en la legislación, pero hubo más notas sobre humo de tabaco que sobre el empaquetado y la publicidad del tabaco.

Al hacer el análisis de las notas por tema relevante, se encontró que la tendencia positiva prevaleció, es decir, el pronunciamiento en general fue a favor del control de tabaco (figura 3). Las notas de tendencia negativa no pasaron de 16\% de todas las notas por tema (38 de 240 en el caso de notas sobre legislación). En el caso de la economía, la mayoría de las notas mostró una tendencia neutra, porque sólo se referían a los gravámenes al producto (tabaco), mismos que impactarían en el bolsillo de la población, ya que el tabaco está considerado, sorprendentemente, en la canasta básica.

La figura 4 muestra si la nota incluye datos duros (estadísticas o datos oficiales) o cita estudios científicos

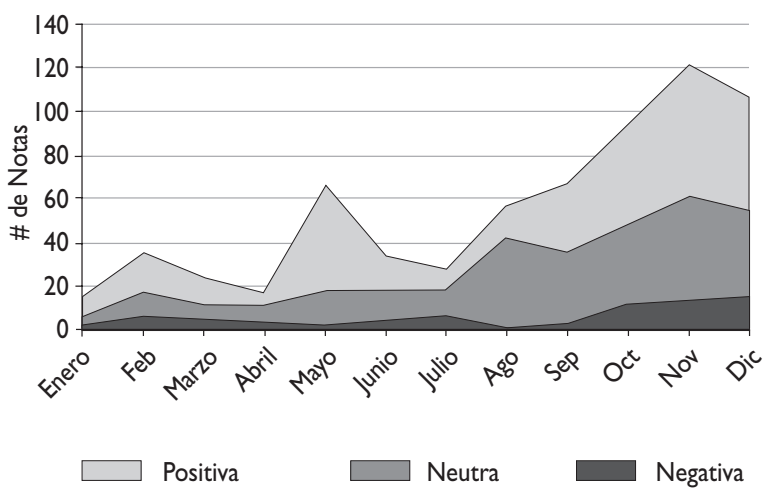

Figura I. Tendencias de las notas sobre tabaco por MES*

salud pública de méxico / vol.50, suplemento 3 de 2008

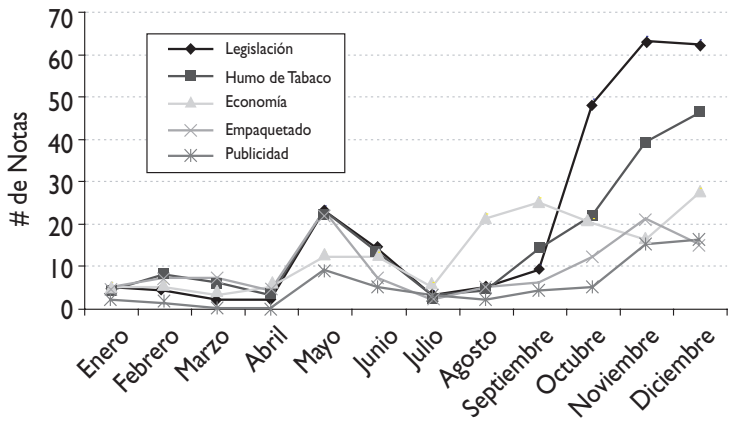

Figura 2. Cobertura de los temas de tabaco por mes

que sustenten la información presentada. Cuando se mencionan datos duros, se indicó si los datos pertenecen a México, a otros países o a México y otros países. Se encontró que $60 \%$ (397 de 660) de las notas sobre tabaco no incluyeron datos duros. Este porcentaje también aplica para las notas sobre legislación (61\%) y economía (63\%). Para los temas humo de tabaco, empaquetado y publicidad, la mayoría de las notas presentaron datos duros y/o evidencias de la investigación científica. Por otra parte, hubo más notas que usaron mayormente o exclusivamente datos mexicanos (19\% solamente datos mexicanos y $5 \%$ datos mexicanos y de otros países), que datos exclusivamente de otros países (16\%); el mayor uso de datos mexicanos se vio reflejado en casi todos los temas (excepto publicidad).

El cuadro II muestra los actores que aparecen en las notas. Los actores con más menciones fueron instituciones gubernamentales (24\%), legisladores (21\%) y tabacaleras $(18 \%)$, a diferencia de las ONG nacional (7\%) e internacional $(8 \%)$; patrón que se repite en los temas de interés. Sobre la publicidad, la presencia de las tabacaleras se elevó hasta $63 \%$ a diferencia del tema del empaquetado, en donde su presencia baja a 30 por ciento.

\section{Conclusiones}

Se destacan algunos hallazgos del presente análisis de la cobertura del tema de tabaco en algunos los medios impresos de comunicación en México. En general, las notas tuvieron una tendencia a favor del control de tabaco $(49 \%)$ o neutra al respecto $(41 \%)$, y solamente una nota de cada 10 tuvo tendencia en contra del control de tabaco. Los y las promotores del Día Mundial sin Tabaco de la OMS lograron cierto éxito en México, porque aumentaron la cantidad de notas sobre el tabaco y el mayor porcentaje de éstas a favor del control de tabaco. 


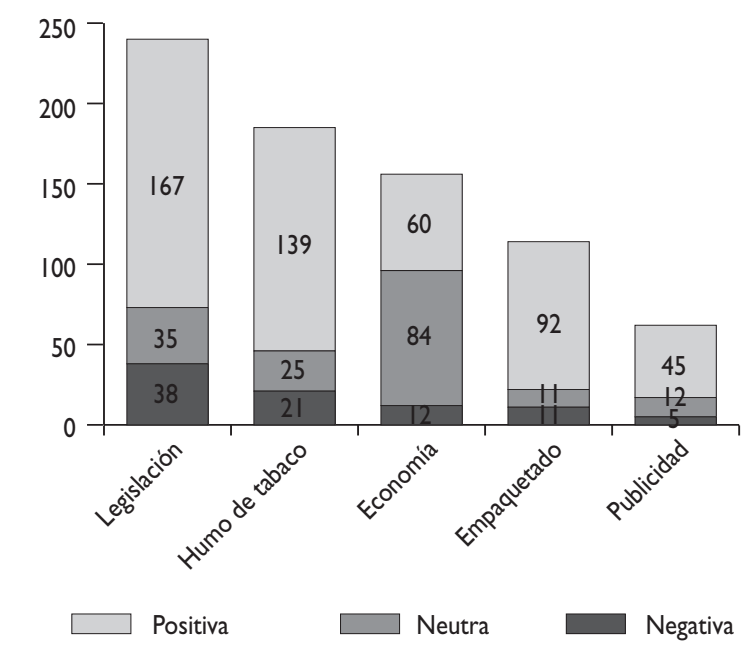

* Los números indican el número de notas por tendencia

Figura 3. Tendencia de las notas sobre tabaco por TEMA.* MÉxICo, 2007

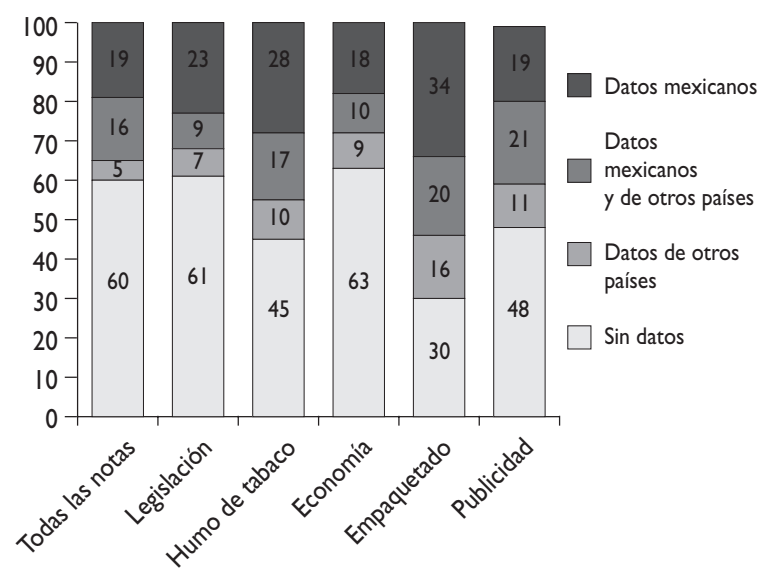

* Los números indican el número de notas utilizando datos de cada fuente

Figura 4. Datos con soporte científico usados en las Notas SObre tabaco POR tema. MéXICO, 207

Cuadro II

ACtORES MENCIONADOS POR TEMA

\begin{tabular}{|c|c|c|c|c|c|c|}
\hline Actores & $\begin{array}{l}\text { Legislación } \\
(n=240)\end{array}$ & $\begin{array}{l}\text { Humo de tabaco } \\
\qquad(\mathrm{n}=185)\end{array}$ & $\begin{array}{c}\text { Economía } \\
(n=156)\end{array}$ & $\begin{array}{c}\text { Empaquetado } \\
\quad(n=1 \mid 4)\end{array}$ & $\begin{array}{c}\text { Publicidad } \\
(n=62)\end{array}$ & $\begin{array}{l}\text { Todas las notas } \\
\qquad(n=660)\end{array}$ \\
\hline Instituciones gubernamentales & $39 \%$ & $38 \%$ & $30 \%$ & $46 \%$ & $48 \%$ & $24 \%$ \\
\hline Legisladores & $50 \%$ & $36 \%$ & $29 \%$ & $34 \%$ & $55 \%$ & $21 \%$ \\
\hline Centros académicos & $9 \%$ & $19 \%$ & $13 \%$ & $32 \%$ & $10 \%$ & $13 \%$ \\
\hline ONG nacional & $16 \%$ & $17 \%$ & $6 \%$ & $12 \%$ & $13 \%$ & $7 \%$ \\
\hline ONG internacional & $13 \%$ & $17 \%$ & $12 \%$ & $22 \%$ & $26 \%$ & $8 \%$ \\
\hline Industria tabacalera & $32 \%$ & $25 \%$ & $24 \%$ & $30 \%$ & $63 \%$ & $18 \%$ \\
\hline Otras empresas y empresarios & $9 \%$ & $11 \%$ & $6 \%$ & $12 \%$ & $3 \%$ & $8 \%$ \\
\hline
\end{tabular}

En el último semestre de 2007, se dio simultáneamente la discusión de la ampliación de la Ley de Protección a la Salud de los No Fumadores en el DF y la de la Ley General para el Control del Tabaco en el Senado de la República. En ese periodo, el tema de tabaco apareció con más frecuencia, y se incrementó el número de notas en contra del control de tabaco, pero las notas claramente en contra no lograron más de 10\% del total de las notas publicadas.

Es evidente que el tema de la legislación y el Día Mundial sin Tabaco llamaron la atención de los medios, y que éstos, generalmente estuvieron a favor del control del tabaquismo.
Al observar la trayectoria de cobertura al paso del tiempo, hay que pensar nuevas maneras de llamar la atención de los medios para mantener el tema vigente en el discurso público, aun en los periodos cuando se observa bajo nivel de interés (por ejemplo, los meses antes y después del Día Mundial sin Tabaco).

Tanto el número como la tendencia de las notas por tema, pueden indicar el desempeño de los diversos actores sociales que están promoviendo el tema de tabaco en los medios de comunicación. La tendencia a favor de control de tabaco es la meta para ganar la opinión pública, y lo vimos para la mayoría de las notas sobre la legislación, el humo de tabaco, empaquetado 
y publicidad. Por el contrario, las notas sobre la economía no lograron producir esta tendencia positiva. Puede ser útil capacitar a los voceros que hablen de la economía del tabaco que mencionen los impuestos y otras políticas públicas como manera de solucionar el problema de tabaquismo. El hecho de que la tendencia de las notas fuera mayormente positiva o neutra, puede indicar que los medios dieron mucho peso a la voz de las instituciones, que se pronunciaban a favor, y poco a la voz de las tabacaleras y/o a las personas que no estaban de acuerdo en limitar su consumo de tabaco, argumentando violación de libertades; o que estaban realmente a favor de las políticas públicas; o que simplemente acuden a las fuentes más visibles, de mayor peso político y social, y les dan cobertura porque eso es lo más conveniente para ellos. Es necesario un análisis más profundo de los resultados para poder corroborar o desechar alguna o todas las afirmaciones anteriores.

La abogacía contempla el uso de datos locales para llamar la atención de los medios de comunicación por la afinidad que puede encontrar la audiencia en ellos. $3,6,7$ Nuestros resultados confirman esta tendencia, al encontrar más notas que usaron datos mexicanos (24\%) que aquellas que usaron datos exclusivamente de otros países $(16 \%)$. Esto nos permite inferir que existe aún espacio para mejorar la difusión de los datos nacionales. Por otro lado, el hecho de que $50 \%$ o más de las notas no se apoyen en datos puede indicar la falta de acceso a la información por parte de los periodistas o la dificultad para utilizarla e interpretarla. En este sentido, los esfuerzos para robustecer la abogacía en medios, deberían fortalecer la capacidad de investigadores, funcionarios e instituciones, quienes generan los datos, para expresarlos en un lenguaje coloquial, y fortalecer las habilidades de los periodistas para tener acceso a estos datos e interpretarlos fácilmente.

Varios actores aparecieron en las notas sobre tabaco, pero los más presentes fueron de instituciones gubernamentales, legisladores y las tabacaleras. Las tabacaleras elevaron su presencia cuando se habló de la publicidad de su producto $(63 \%)$, que podría reflejar el gran interés de esta industria en no restringir la comercialización y mercadotecnia de su producto. La presencia abrumadora de actores gubernamentales por encima de las ONG sugiere que la actividad de abogacía de la sociedad civil en los medios masivos aún no hacía explosión al finalizar 2007. Para incrementar la presencia de las ONG nacionales frente a los medios, puede ser importante construir coaliciones con sectores de la sociedad civil (por ejemplo, padres y madres de familia, derechos humanos y sexuales) que hasta ahora no han estado involucrados en el tema de tabaco en México. ${ }^{20}$

El presente estudio tiene algunas limitaciones. Entre ellas está la falta de análisis de los medios electrónicos de comunicación (por ejemplo, televisión, radio, internet), lo que se pretende hacer en el futuro para determinar la coincidencia entre los medios impresos y los electrónicos. Tampoco se analizaron las notas del primer trimestre de 2008, justo cuando la legislación del DF se modificó para ser más fuerte, entró en vigor, y cuando el Senado aprobó la ley federal. Será importante entender cualquier cambio de tendencia y de cobertura durante y después de estos eventos. Hace falta más investigación cualitativa de la cobertura del tema en los medios sobre el cumplimiento de la ley, pero sobre todo, sobre la difusión de información fidedigna, para lograr que la población en general tome decisiones informadas.

Hace falta más trabajo con y para los medios, para formarlos y tal vez especializarlos en dicha cobertura. El presente estudio nos indica que los medios de comunicación ya están a favor del control de tabaco o, por lo menos, lo representan de manera neutra. La abogacía para promover las políticas públicas del control de tabaco debe reconocer estos aliados valiosos en la lucha contra el tabaquismo.

\section{Referencias}

I. McQuail D, Siune K, eds. Media policy: Convergence, concentration and commerce. London: Sage, 1999.

2. Lawson $\mathrm{CH}$. Building the fourth estate: Media opening and democratization in Mexico. In: Middlebrook KJ, ed. Dilemmas of political change in Mexico. London: Institute of Latin American Studies, 2004:373-400.

3. Dorfman L. Using media advocacy to influence policy. In: Cohen L, Chavez V, Chehimi S, eds. Prevention is primary: Strategies for community well being. San Francisco: Jossey-Bass, 2007:181-203.

4. Chapman S, Dominello A. A strategy for increasing news media coverage of tobacco and health in Australia. Health Promot Int 200I; I6:I37-I43.

5. Wallack LM, Themba-Nixon M, Dorfman L, Jernigan D. Media Advocacy and Public Health: Power for prevention. London: Sage, 1993.

6. Chapman S, Lupton D. The fight for public health: Principles \& practice of media advocacy. Oxford: Blackwell BMJ Books, 1994.

7. Efroymson D. Using media and research for advocacy: Low cost ways to increase success. Ottawa, Canada: HealthBridge, 2006.

8. Ashbridge M. Public place restrictions on smoking in Canada: Assessing the role of the state, media, science and public health advocacy. Soc Sci Med 2004;58:13-24.

9. Clegg-Smith K, Wakefield MA, Terry-McElrath Y, Chaloupka FJ, Flay BR, Johnson L, et al. Relation between newspaper coverage of tobacco issues and smoking attitudes and behavior among American teens. Tob Control 2008; 17:17-24.

10. Stillman FA, Cronin KA, Evans WD, Ulasevich A. Can media advocacy influence newspaper coverage of tobacco: Measuring the effectiveness of the American stop smoking intervention study's (ASSIST) media advocacy strategies. Tob Control 200 I; 10:137-I44.

I I. Niederdeppe J, Farrelly MC, Wenter D. Media advocacy, tobacco control policy change and teen smoking in Florida. Tob Control 
2007; $16(I): 47-52$.

12. Dorfman L. Studying the news on public health: How content analysis supports media advocacy. Am J Health Behav 2003;27(supp 3):S2 17-S226. 13. Menashe C, Siegel M. The power of a frame: An analysis of newspaper coverate of tobacco issues-United States, 1985-1996. J Health Commun 1998;3:307-325.

14. Clegg-Smith K, Wakefield M, Edsall E. The good news about smoking: How do US newspapers cover tobacco issues. J Public Health Policy 2006;27:166-18I.

15. Clegg-Smith K, McLeod K, Wakefield M. Australian letters to the editor on tobacco: Triggers, rhetoric and claims of legitimate voice. Qual Health Res 2005; I5(9): I I80-II 98.

16. Clegg-Smith K, Terry-McElrath Y, Wakefield M, Durrant R. Media advocacy and newspaper coverage of tobacco issues: $A$ comparative analysis of I year's print news in the United States and Australia. Nicotine Tob Res 2005;7(2):289-299.

17. Padrón Nacional de Medios Certificados 2007. México: Secretaría de Gobernación, 2007.
18. Clegg-Smith K, Wakefield M, Siebel C, Szczypka G, Slater S, TerryMcElrath Y, et al. Coding the news: The development of a methodological framework for coding and analyzing newspaper coverage of tobacco issues. Chicago: University of Illinois \& The Robert Wood Johnson Foundation, 2002.

19. Hayes AF, Krippendorff K. Answering the call for a standard reliability measure for coding data. Communication Methods and Measures 2007; : :77-89.

20. Thrasher JF, Reynales-Shigematsu L, Baezconde-Garbanati L, Villalobos V, Téllez-Girón P, Arillo-Santillán E, et al. Promoting the Effective Translation of the Framework Convention on Tobacco Control: A Case Study of Challenges and Opportunities for Strategic Communications in Mexico. Eval Health Prof 2008;31:145-166. 